\title{
Software Design and Development of Data Collection and Analysis for Classroom Teaching and Learning Behaviors
}

\author{
Yun Cheng, Lili Zheng, Yanli Wang, and Feng Wang
}

\begin{abstract}
According to the actual needs of collecting and analyzing the classroom teaching and learning behavior, the software is analyzed and designed from three aspects of software architecture, interface and main functions, and its functions of teaching behavior acquisition and encoding, data processing, analysis, and visualization has been realized by technology. The software application result shows that the basic functions of the software are able to realize the normal use, its performance is stable, the anticipated goal is achieved, and meantime it also has the characteristics such as reducing the behavior observation difficulty, simplifying data processing, and data input and export diversification. This will improve the efficiency and quality of classroom teaching and leaning behavior observation, so that researchers can focus on the behavior analysis. It has great significance and value for promoting teachers' professional development and improving the quality of education and teaching.
\end{abstract}

Index Terms-Classroom teaching, classroom teaching and learning behaviors, classroom observation, behavior analysis.

\section{INTRODUCTION}

In China, classroom instruction is the main form of formal education, and classroom teaching is the main channel for students' development. Under the background of educational informationization, using information technology to innovate the analytical method of classroom teaching and learning behaviors (hereafter referred to as simply CT\&LB) has important significance. Through the development and application of data collection and analysis for CT\&LB, using technology to reduce the application threshold of teaching analysis and evaluation methods, to improve the efficiency of data collection and analysis, It is conducive to the promotion and application of teaching analysis and evaluation methods, it can improve teachers' ability of teaching, analysis and evaluation, effectively promote the development of teaching

Manuscript received August 12, 2017; revised November 25, 2017. This research is supported by the National Natural Science Foundation of China (Grant No.71603098), the research program of Hubei province education department (Grant No. Q20132905), the key project of 12th five-year plan of education science of Hubei province (Grant No. 2014A036), humanities-society scientific research program of Hubei province education department (Grant No.15Q238, 17Q184), the teaching and research project of HuangGang Normal University (Grant No. 2017CE07).

Yun Cheng and Yanli Wang are with School of Educational Science and Technology, HuangGang Normal University, Hubei, China (corresponding author: Yanli Wang; e-mail: yunlccnu@126.com,wylccnu@126.com).

Lili zheng is with HuangGang Normal University, Hubei, China (e-mail: 1284732486@qq.com).

Feng Wang is with Organization Department, HuangGang Normal University, Hubei, China (e-mail: wangfeng@hgnu.edu.cn). activities, and improve the teaching efficiency and quality.

\section{REQUiREMENT ANALYSIS OF SOFTWARE DESIGN}

The research of classroom teaching behavior has been the focus of research in the educational field. By introducing the technologies support, the related scholars carry out the classroom teaching analysis and evaluation research supported by technology. Though there are some influential classroom teaching tools in foreign countries, such as 'Transana' [1], 'QSR Nvivo' [2], 'Atlas.ti' [3] and so on, these tools are not free, and the uses of these software is complex, It needs professional knowledge to collect and analyze the CT\&LB, some adverse effects is brought in application and popularization.

On the basis of comparative analysis of existing related software, combined with actual demand, the Software of data collection and analysis for CT\&LB should have the following characteristics: The interface of this software was terse, friendly and can be used flexibly and conveniently with steady and safe in motion, with the function of data collection, storage, analysis and presentation for CT\&LB.

\section{SOFTWARE DESIGN}

\section{A. Software Architecture Design}

Based on classroom teaching video, the analysis software of CT\&LB can collect, encode, store, analyze and visualize the data of CT\&LB. According to the basic requirements, the software is mainly divided into three layers: Application layer, Business Logic Layer and Data service layer.

The application layer is directly oriented to the user, mainly providing an interactive interface between the user and the software. The user interacts with the software through the application layer, calls the software functions such as teaching observation, the data collection of CT\&LB, data display, analysis and evaluation, send a request to the corresponding processing module, and the application layer will be the final processing results back to the user.

The business logic layer is used in response to the user application layer transfer request, and calls the relevant logic processing function, processing the users' requests, including data storage, data retrieval, data analysis, data export, etc.

The data service layer mainly provides data support services, including the data of CT\&LB, the data generated during the analysis of CT\&LB and so on.

\section{B. Software Interface Structure Layout Design}

The software interface consists of seven parts: title bar, menu bar, the control area for video playback, the area for 
basic information settings of classroom teaching, the area for data collection and coding for CT\&LB, the control area for coding, the area of copyright information, and the layout is shown in Fig. 1.

\begin{tabular}{|c|c|}
\hline \multicolumn{2}{|c|}{ Title bar } \\
\hline \multicolumn{2}{|c|}{ Menu bar } \\
\hline $\begin{array}{c}\text { The control area for video } \\
\text { playback }\end{array}$ & $\begin{array}{l}\text { The area for basic information } \\
\text { settings of classroom teaching }\end{array}$ \\
\hline $\begin{array}{c}\text { The area for data collection and } \\
\text { coding for CT\&LB }\end{array}$ & The control area for coding \\
\hline \multicolumn{2}{|c|}{ The area of copyright information } \\
\hline
\end{tabular}

Fig. 1. Layout design of the software interface.

\section{Main Function Module Design}

According To Actual Demand for analyzing of CT\&LB, the software provides three core functional modules: Data collection and coding of CT\&LB, Data processing and analysis of CT\&LB, Visual presentation of data of CT\&LB, and the main function module design is shown in Fig. 2.

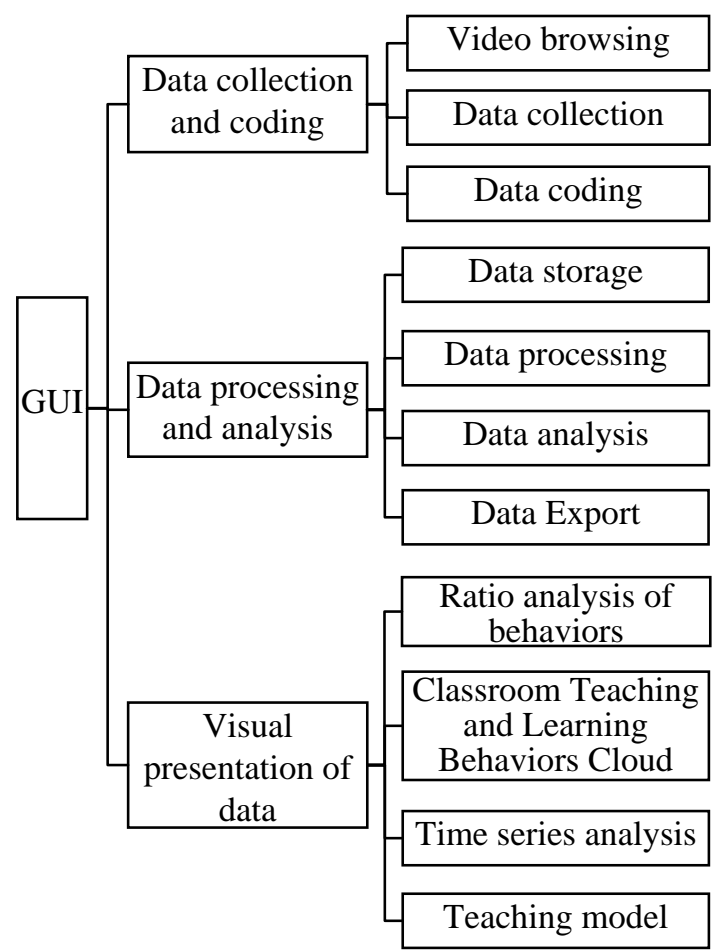

Fig. 2. The main function module design.

\section{SOFTWARE DEVELOPMENT}

\section{A. Software Development Environment}

The operating system for developing this software is Windows 7 Ultimate edition Sp1, the main development tool is Microsoft Visual Studio 2010, the Framework version is 4.0, and the development language is $\mathrm{C \#}$.

\section{B. Data Collection and Coding}

The user interface effects of the software as is shown in Fig. 3. In order to facilitate the operation, the software sets the accessible tools, such as Play, Pause, and so on. And an operation button is set for each type of teaching behavior. If the observer needs to record the data of some behavior, all he had to do was to click the corresponding button; the software will automatically record the current time and behavior type, and completes the encoding. The observer completed behavioral observation and data collection; he can select the function menu according to the requirement, save or analysis the data.

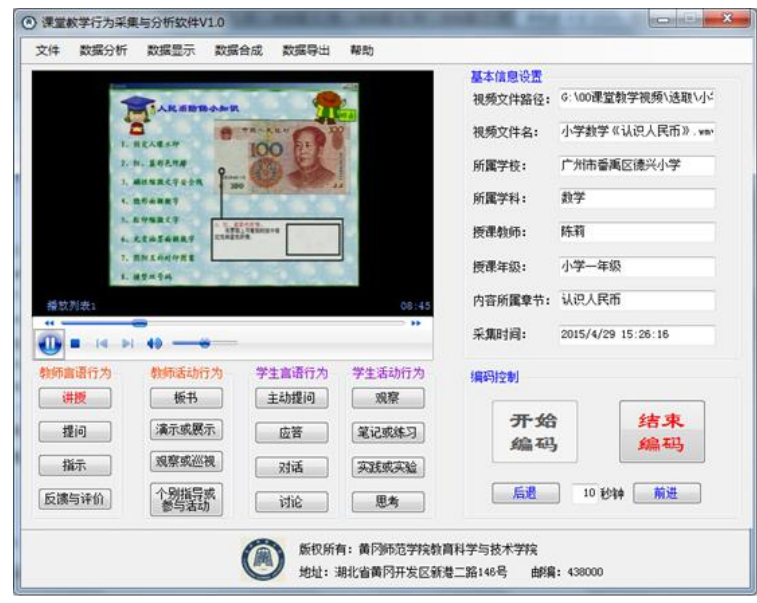

Fig. 3. The interface for data collection of CT\&LB.

\section{Data Processing and Analysis}

1) Data storage of CT\&LB

The storage of the encoding data follows the Extensible Markup Language (XML) specification, the encoding data of the classroom teaching video is stored in a XML file [4]. The XML file contains all the logical slice of video information, such as the information of the video file, the sample id, current time, and the type of behaviors. The format of the XML file is shown below:

$<$ ?xmlversion $=$ "1.0" eneoding $=$ "UTF-8" $>$

$<$ !--this is Teaching and Learning Behavior in Classroom-->

$<$ lesson videoFile $="$ File name" duration $="$ the length of the video "fileType $=$ " File Format "fileSize=" File Size " school="school name" subject= "subject" teacher= "teacher name" grade= "grade" chapter = "Teaching contents" date $=$ "date" $>$

<behaviors>

$\langle$ behavior id $=" 1 ">$

$<$ currentTime $>2</$ currentTime $>$

$<$ behaviorType $>1</$ behaviorType $>$

$</$ behavior $>$

$<$ behavior id="2">

$<$ currentTime $>4</$ currentTime $>$

$<$ behaviorType $>2</$ behaviorType $>$

$</$ behavior $>$

.......

$</$ behaviors $>$

</lesson>

The file presents 2 sample data of CT\&LB, each sample data corresponds to a behavior node of the XML file, and the fragments between the two sample form a logical fragment of the video. Each behavior node has an ID attribute which means the number of sample; It has two child nodes, one child node(currentTime) represents the specific time at which the sample is located(in seconds), the other child 
node(behaviorType) represents the CT\&LB categories of data encoding of the sample.

2) Data processing of CT\&LB

The sample of teaching behavior should contain three kinds information: subject of behavior, category and time. Subject of behavior refers to the subject of CT\&LB (teacher or student); Category refers to the specific teaching behavior category of sample (behavior categories in a coding system for CT\&LB); time refers to the CT\&LB occurs at the moment (the sample time of the sample of CT\&LB).

If data analysis is performed directly with the above data, we can only get the basic information such as number of sample, sampling time and so on. So, it is necessary to sort out the sample information, and get the specific duration information for each behavior for further analysis.

As the sample time is stored in each sample information, so, The difference between the sample time of the two adjacent samples is the duration of the previous sample, and here is an example: The sample time of $\mathrm{M}$ behavior is 51 second, The sample time of next $\mathrm{N}$ behavior of adjacent is 86 second, The difference between the sample time of the two adjacent samples is 35 seconds, and it is the duration of $\mathrm{M}$ behavior.

3) Analysis of CT\&LB

Based on the data of CT\&LB, we can Analysis the behavior in many ways, such as Behavioral occupancy analysis, variance analysis, $\mathrm{Z}$ score analysis, Multilevel scoring [5], Classroom Teaching and Learning Behaviors Cloud Analysis [6], Teaching Modes Analysis, it can provide the basis for the analysis and evaluation of CT\&LB.

4) Data exporting of CT\&LB

According to users' requirements, the relevant sample data and analysis data of CT\&LB in the software can be exported and stored as Excel, TXT and other common formats.

\section{Visual Presentation of Data}

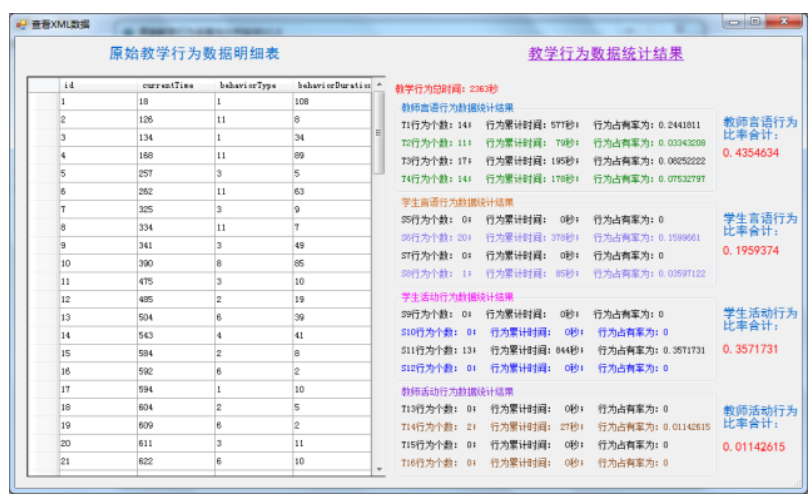

Fig. 4. The essential information statistics example of CT\&LB

For users, they do not need to know the details of the data processing and analysis process, but concern about the results of data processing. If the analysis data is presented directly to the users, it can not directly reflect the specific circumstances of the teaching behaviors. For example, if the score of every kind of teaching behaviors is shown directly to the users, they do not have a direct and clear distribution of the teaching and learning behaviors cloud in their minds, and just give the subjective judgment on the overall situation (such as the instructional mode) according to the data. Therefore, the analyzed data of the teaching and learning behaviors should be visualized. The following forms can be used for data display, such as data statistics, Time series analysis of CT\&LB, Classroom Teaching and Learning Behaviors Cloud Analysis, Instructional Modes Analysis, as shown in Fig. 4 to Fig. 7.

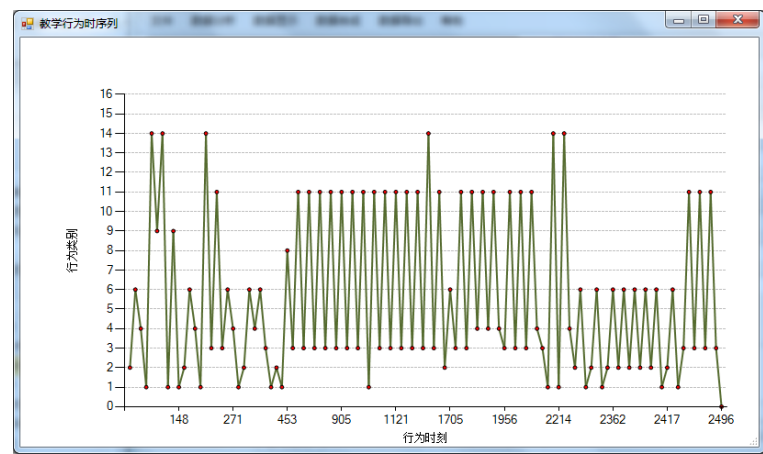

Fig. 5. Time series analysis example of CT\&LB.

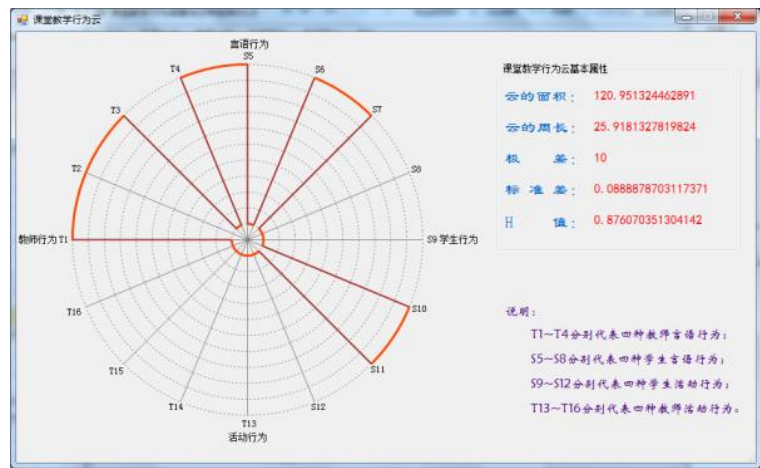

Fig. 6. The Cloud Analysis example of CT\&LB.

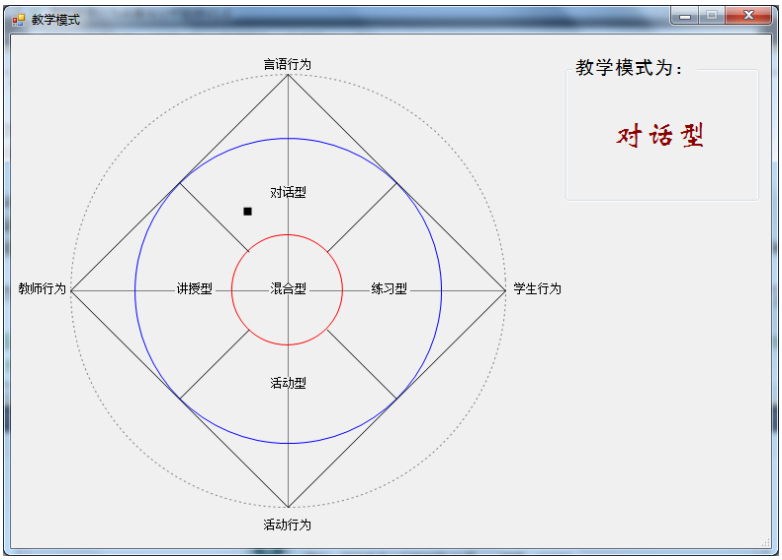

Fig. 7. The example of instructional modes analysis.

\section{ApPliCATION AND RESUlt}

After the software has been developed, we tested its function and performance, and applied it to analyze several classroom teaching videos selected carefully. The application result shows that the basic functions of the software are able to realize the normal use and its performance is stable, the anticipated goal is achieved, meantime it also has the following characteristics.

1) Reduce the difficulty of observing the classroom teaching and learning behaviors. Provide a variety of ways to accurately position the teaching content, so we just need to click on the corresponding button that it can automatically realize the data acquisition and encoding, greatly reducing the difficulty of observing classroom teaching behaviors, improving the accuracy and 
efficiency of data collection.

2) Simplify data processing. The software can automatically count and analyze the data of teaching and learning behaviors, and present the result in a variety of ways; users can analyze and interpret it as needed.

3) Data input and export diversification. Data input can be based on video capture, or you can import existing data directly. Teaching behavior collection and coding data are stored in a XML file. According to the analysis requirements, it can be automatically translated into FIAS, $\mathrm{S}-\mathrm{T}$ and other behavioral sequences. The teaching behavior collection and analysis data can be exported to Txt, Excel and other formats.

\section{CONCLUSION}

Teachers' classroom observation and analysis of classroom teaching activities will help teachers' professional development, so as to effectively improve the quality of education and teaching, and students will also get more promotion [7]. The study has explored the observation methods of classroom teaching and learning behaviors under the support of technology, and designed auxiliary software, in order to reduce the difficulty and improve the efficiency and quality of observing classroom teaching and learning behaviors, so that researchers will focus on classroom behavior analysis. It has great significance and value for promoting teachers' professional development and improving the quality of education and teaching.

\section{REFERENCES}

[1] Transana[EB/OL]. [Online]. Available: http://www.transana.org

[2] QSR Nvivo[EB/OL]. [Online]. Available: http://www.qsrinternational.com

[3] Atlas.ti [EB/OL]. [Online]. Available: http://atlasti.com

[4] (2006). W3C.extensible markup language. [Online]. Available: http://www.w3.org/XML/

[5] D. Fu et al., Education Information Processing (2nd Edition) [M], Beijing: Beijing Normal University Press, 2011.
[6] Y. Cheng et al., "The research on construction and application of cloud model for analysis of classroom teaching and learning behaviors [J]," Journal of Distance Education, 2017, vol. 02, pp. 36-42.

[7] Y. Zhan et al., "Inciting the social imagination: Education research for the public good: Perspective from2011 AERA annual meeting [J]," Journal of Distance Education, 2011, vol. 5, pp. 3-14.

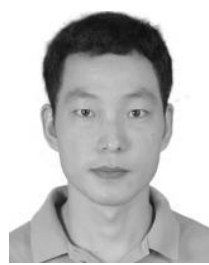

Yun Cheng is an associate professor in HuangGang Normal University, Huanggang, Hubei province, China. He was born in 1982, in Xiangyang, China. He studied educational technology in Central China Normal University from 2001-2008, and received his bachelor's degree \& master's degree. Next, he worked in HuangGang Normal University as a teacher since June 2008. Then worked towards a $\mathrm{PhD}$ degree in Central China Normal University with the major of educational technology, and earned his doctor's degree in 2015 . He is currently working at school of educational science and technology, HuangGang Normal University, and his research interests is focused on learning analysis technology.

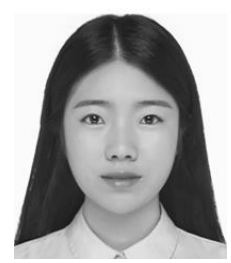

Lili Zheng was born in 1993, in Juzhou, Zhejiang Province, China. She studied modern educational technology in HuangGang Normal University Since 2017, She is a graduate student at HuangGang Normal University, Huanggang, Hubei province, China, her research interests is focused on networking and new media applications.

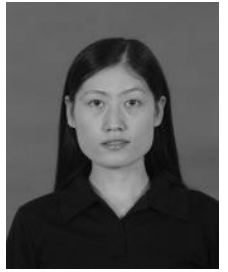

Yanli Wang was born in 1982, in Xiangyang, China She studied educational technology in Central China Normal University from 2001-2008, and received her bachelor's degree \& master's degree. Then she is currently working at school of educational science and technology, HuangGang Normal University, Huanggang, China, her research interests is focused on basic theory, method and application of educational technology.

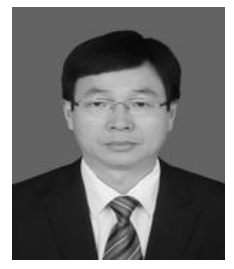

Feng Wang is a professor in HuangGang Normal University (China). He was born in 1964 in Yingshan, China. He graduated from Huazhong University of Science and Technology, and earned his doctor's degree in 2006. He studied information and communication engineering, and his research interests are focused on application of modern educational technology, Cooperative communication technology. 